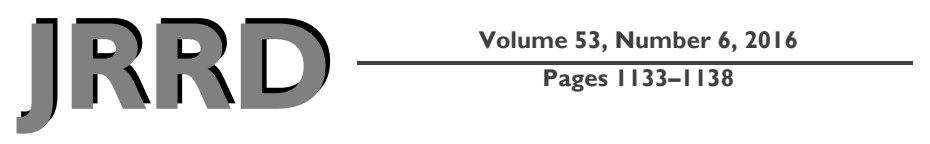

\title{
Transtibial amputee gait efficiency: Energy storage and return versus solid ankle cushioned heel prosthetic feet
}

\author{
James Gardiner, PhD; ${ }^{*}$ Abu Zeeshan Bari, PhD; ${ }^{2}$ David Howard, PhD; ${ }^{1}$ Laurence Kenney, PhD $^{\mathbf{2}}$ \\ ${ }^{1}$ School of Computing, Science, and Engineering and ${ }^{2}$ School of Health Sciences, University of Salford, Salford, UK
}

\begin{abstract}
Energy storage and return (ESR) feet have long been assumed to promote metabolically efficient amputee gait. However, despite being prescribed for approximately $30 \mathrm{yr}$, there is limited evidence that they achieve this desired function. Here, we report a meta-analysis of data from 10 studies that met our selection criteria to determine whether amputee walking with ESR feet is more efficient than with conventional solid ankle cushioned heel (SACH) feet. Additionally, the data were tested for a relationship with walking speed since it has been suggested ESR feet might perform better at higher speeds. The raw data were highly variable because of differences in study protocols; therefore, we normalized the data and found a statistically significant difference $(p<0.001)$ between ESR and SACH feet. However, the magnitude of this difference is small, with the cost of transport (COT) with ESR feet being $97.3 \%$ of the cost with $\mathrm{SACH}$ feet. No relationship between normalized ESR COT and speed was found ( $p=$ 0.19 ). We hypothesize that the small but statistically significant difference between ESR and SACH feet may not constitute a functionally significant improvement in COT, possibly related to the limited push-off power provided by ESR feet compared with nondisabled ankles.
\end{abstract}

Key words: amputee, cost of transport, energy storage and return, feet, gait, prosthetics, rehabilitation, $\mathrm{SACH}$, transtibial, walking.

\section{INTRODUCTION}

The negative effect of lower-limb amputation on quality of life is significant [1-2] and in part caused by the poor energy efficiency of amputee gait. Compared with that of nondisabled subjects, the gait of individuals with amputation is more costly per minute and per meter [3-6]; the latter often referred to as cost of transport (COT). Furthermore, the higher up the leg the amputation, the higher the COT becomes [5,7]. Over approximately three decades, engineers have designed prosthetic feet consisting of one or more elastic blades (Figure 1(a)), which deform during early stance and recoil in late stance. It has long been assumed that these feet help to reduce amputee COT, especially when compared with traditional solid ankle cushioned heel (SACH) feet. Collectively, many of these designs are referred to as energy storage and return (ESR) feet. ESR feet are thought to replicate one of the functions of the Achilles tendon and associated leg musculature, storing and releasing strain energy during walking [8-11].

Unfortunately, published data have not led to a consensus on whether ESR feet do indeed reduce amputee COT. Some articles have found a statistically significant, albeit small, improvement in COT with ESR versus SACH feet [12-13]. Most studies, however, have failed to demonstrate any statistically significant differences $[3,4,6,14-18]$. In the most recent systematic review of the literature, Hofstad et al.'s Cochrane review found "a

\footnotetext{
Abbreviations: $\mathrm{COT}=$ cost of transport, $\mathrm{ESR}=$ energy storage and return, $\mathrm{SACH}=$ solid ankle cushioned heel.

*Address all correspondence to James Gardiner, PhD; PO33 Blatchford Bldg, University of Salford, M6 6PU UK; +44-161-295-6429. Email: j.d.gardiner@salford.ac.uk http://dx.doi.org/10.1682/JRRD.2015.04.0066
} 


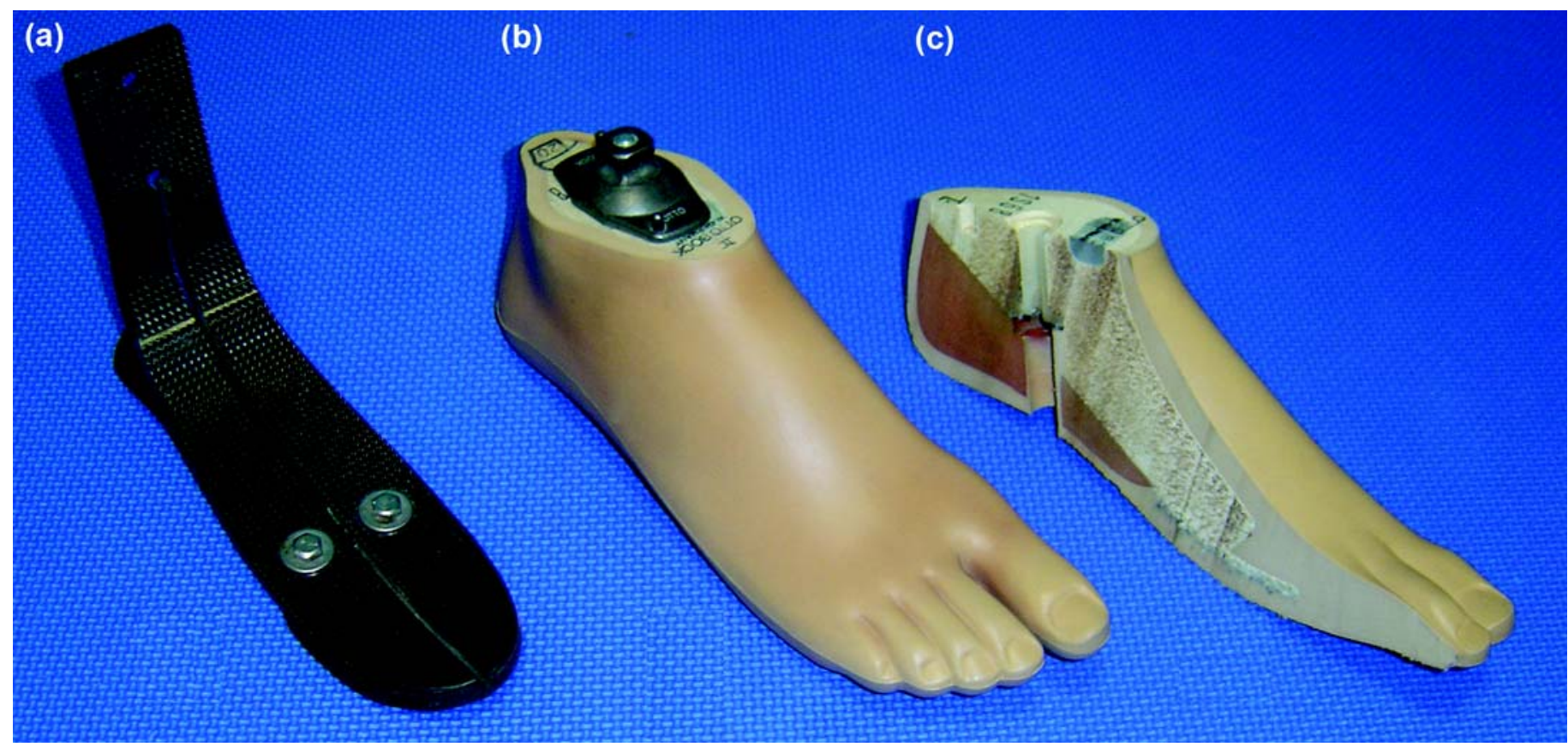

\section{Figure 1.}

(a) A typical energy storage and return foot, showing the blades designed to store strain energy during stance and release it again at push-off. (b) Conventional solid ankle cushioned heel $(\mathrm{SACH})$ foot. (c) Cut-through of SACH foot showing the wooden keel beneath the outer cover and the significant cushioning in the heel section.

small trend towards the Flex-foot (a type of ESR foot) in comparison with the SACH foot for . . . lower energy cost in individuals with a transtibial amputation" [19]. However, unusually for a Cochrane systematic review, this conclusion was not based on a meta-analysis of the data.

Few studies comparing amputee COT using different feet include a nondisabled control group, which makes comparison of results from different studies difficult because of potential protocol bias. A solution to this problem is to use an alternative method of normalizing the data, thereby allowing meaningful comparisons between studies. Since many studies of amputee walking include conventional SACH feet, these results can be used to normalize the results for all other feet types. Here, we present a novel meta-analysis of articles identified in Hofstad et al.'s systematic review [19] comparing ESR feet with SACH feet to determine whether the hypothesized improvement in COT is evident in the combined data from published studies. Additionally, we analyzed the data to see whether there was a relationship with walking speed since it is thought that ESR feet may be more effective at higher speeds $[3,12]$.

\section{METHODS}

Applicable studies for our meta-analysis were identified from the most recent systematic review of the literature [19]. Note that we are not attempting to repeat the systematic review, since this has already been conducted and is maintained by the Cochrane Collaboration, with the most recent search being conducted in April 2006 and Hofstad et al.'s [19] article updated. Rather, we are building upon the review article's foundation and conducting a meta-analysis of the data contained within articles already judged to be of sufficient quality to be included in Hofstad et al.'s review. Not all articles contained within the systematic review were suitable for our meta-analysis, and therefore we applied four additional criteria in addition to the criteria used in Hofstad et al.'s Cochrane review [19]:

1. We included only studies on subjects with unilateral transtibial amputation to avoid the possible confounding influence of prosthetic knee joints.

2. We excluded studies involving subjects with vascular amputation to avoid the possible confounding influences 
of their increased cost of walking, additional health problems, and reduced fitness [17-18,20].

3. We required the studies to include $\mathrm{SACH}$ feet and also devices that are commonly referred to as ESR feet to enable our normalization approach.

4. We required the studies to contain metabolic cost of walking data, either per minute or per meter.

After the application of the four additional criteria, we identified nine articles [3,4,12-18] from Hofstad et al.'s [19] systematic review that contained the necessary data for our meta-analysis. In addition, we identified only one article (Mengelkoch et al. [6]) published after the systematic review that contained the relevant data and met the necessary selection criteria for the normalization method. The additional article (Mengelkoch et al. [6]) met Hofstad et al.'s quality criteria and therefore was also included in our data set.

If the data from an included study were presented as cost of walking per minute, then these results were divided by walking speed to provide COT (i.e., cost per meter). To normalize the data, we divided the ESR COT by the SACH COT from the same study at each recorded speed; this value will be referred to as the normalized ESR COT. In essence, normalized ESR COT is the fraction of ESR COT versus SACH COT at every speed within the study. Only one study, Torburn et al. [17], did not include exactly identical speeds for SACH and ESR feet, because they were self-selected speeds. However, all these speeds were very closely matched (range from 81.0 to $84.7 \mathrm{~m} / \mathrm{min}$ ), and therefore the data were still considered suitable for our normalization method. A linear relationship between normalized ESR COT and walking speed was determined using an ordinary least squares method regression analysis. The mean normalized ESR COT was compared against the null hypothesis mean of 1 (i.e., no difference between ESR and SACH) using a Wilcoxon signed-rank test because the Kolmogrov-Smirnov test for normality showed the data were not normally distributed $(p<0.001)$. All data analyses were performed using MATLAB R2014b with the statistics toolbox (The MathWorks, Inc; Natick, Massachusetts).

\section{RESULTS}

Data were collected from 10 studies (Appendix). The raw data (Figure 2(a)) shows the variation in the COT measurements reported. The difficulty in drawing any con-
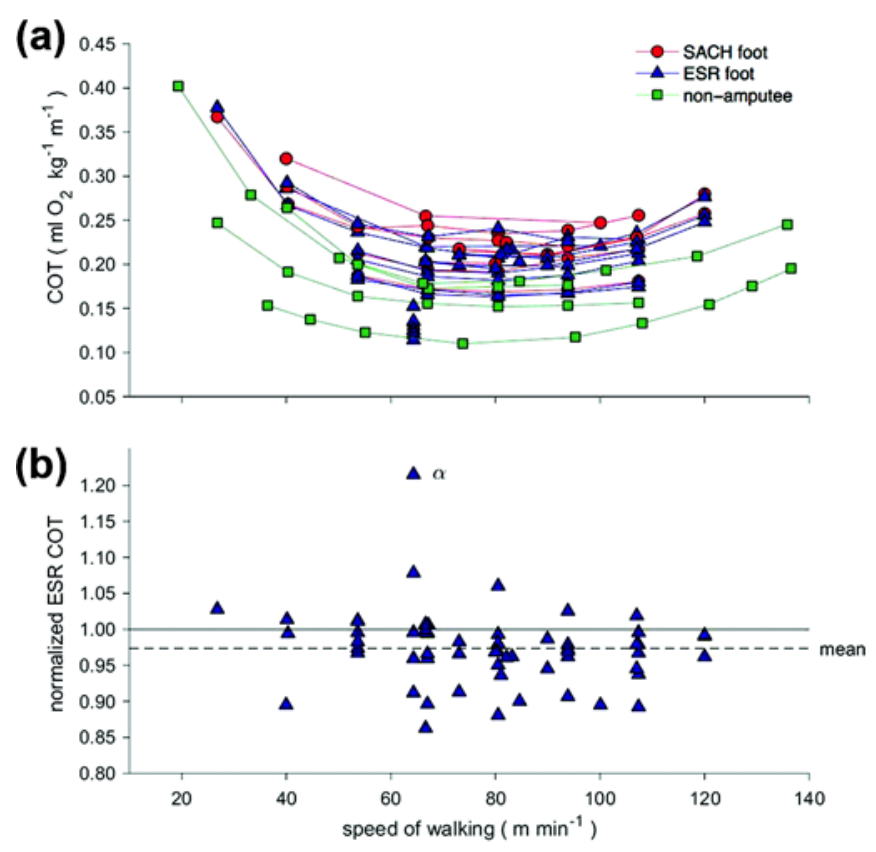

Figure 2.

Cost of transport (COT) versus walking speed gathered from the literature for subjects with nonvascular, transtibial amputation.

(a) Raw data for solid ankle cushioned heel (SACH) feet (circles) and energy storage and return (ESR) feet (triangles). Nonamputee data (squares) are included for comparative purposes. The overlap and variability of the raw data, caused by protocol differences, highlights the difficulties in understanding the effect of ESR feet and, hence, the requirement to normalize the data. (b) ESR COT normalized by SACH COT from the same study at each recorded speed (termed normalized ESR COT). Mean normalized ESR COT is 0.973 (dashed line), which is significantly lower (Wilcoxon signed-rank test) than the expected mean of 1 (solid line) if no difference existed. An ordinary least squares method regression found no significant relationship between normalized ESR COT and speed of walking. Removing the outlying point ( $\alpha$ ) from the analysis changed the mean normalized ESR COT from 0.973 to 0.969 . Therefore, the point was kept in the analysis since it had little effect on the overall discussion of the results.

clusions from the raw data is clear and highlights the importance of normalizing the data. Some data for control walking are included for reference [4-6,21]. The raw COT data (Figure 2(a)) is roughly U-shaped, with an optimum walking speed around $80 \mathrm{~m} / \mathrm{min}$. However, Figure 2(b) shows that the U-shape disappears after normalizing the 
ESR COT with respect to the SACH COT. The normalized ESR COT data have a mean of 0.973 that is significantly different from 1 , the null hypothesis mean $(Z=-4.582, p<$ $0.001)$. The normalized ESR COT was shown by the regression analysis to be independent of the speed of walking $\left(F_{1,58}=1.753, r^{2}=0.029, p=0.19\right)$. Removing the potentially outlying point ( $\alpha$; Figure 2(b)) from the analysis changed the mean normalized ESR COT to 0.969, which has little effect on the overall interpretation and discussion of the results. Therefore, the outlier $(\alpha)$ was kept in for the entire analysis.

\section{DISCUSSION}

The results presented here provide the first conclusive evidence that ESR feet reduce the cost of walking (per meter) in subjects with amputation versus conventional SACH feet. However, this reduction is very small, with the mean cost of amputee walking across all ESR feet being 97.3 percent of that with a SACH foot. This confirms what only a few studies have shown with statistical significance $(p<0.05)[12-13]$. The majority of studies have failed to obtain statistically significant results [3-4,6,14-18], probably because of insufficient statistical power to detect such small differences. The lack of a relationship between speed and normalized ESR COT (Figure 2(b)) was unexpected because it has previously been suggested that ESR feet may only be effective compared with $\mathrm{SACH}$ feet at higher walking speeds $[3,12]$. It is interesting to note that the apparent clustering of the 10 lowest points in the normalized ESR COT analysis (Figure 2(b)) is based on data from six different studies [4,12-13,15,17-18] and six different ESR foot designs. Therefore, this apparent clustering of the data is not due to the protocols of a few studies or feet that have consistently different designs from the rest of the feet.

Studies of amputee gait highlight the fact that passive prosthetic feet of all types (including ESR feet) provide a small fraction of the push-off power generated by an intact foot and ankle [22-24]. The reduced push-off power of passive ESR feet is likely because the neutral (zero moment) ankle angle of a passive prosthesis corresponds to normal standing, which means that once the prosthesis moves into plantar flexion, it will always produce a dorsiflexion moment (acting to return it to neutral), the opposite of what is required during push-off. This greatly reduces push-off at the end of stance com- pared with nondisabled intact ankles [23-24]. As a result, subjects with amputation using ESR feet have to compensate for the lack of push-off power using more proximal joints and their sound limb. We believe that poor push-off is a likely cause of the disappointingly small improvement in COT achieved by passive ESR feet versus $\mathrm{SACH}$ feet. It is important to note that, although the majority of studies included in our meta-analysis are based on relatively older feet, modern ESR feet are still broadly based on the same design and therefore lack the ability to plantar flex beyond neutral during push-off. Indeed, people with amputation who use an actively driven prosthetic foot and ankle show a reduction in COT compared with those using ESR feet; furthermore, no statistically significant difference in their COT compared with nondisabled controls has been found [25]. Additionally De Asha et al. suggested that reducing the "braking effect" that many prosthetic feet produce at the start of stance may also be important for improving prosthetic ankle/foot function and reducing the metabolic cost of gait for those with amputation [26]. In conclusion, despite significant effort to develop more energy efficient passive prosthetic feet, the results are disappointing. While our meta-analysis of normalized COT during level walking of individuals with nonvascular, unilateral, transtibial amputation is not a comprehensive comparison of ESR and conventional SACH feet, we feel these findings support the view that further research is required on the design of passive prostheses as well as on actively controlled and powered prosthetic feet (for review see Versluys et al. [27]).

\section{CONCLUSIONS}

ESR feet only marginally improve the COT in subjects with traumatic transtibial amputation compared with SACH feet. In our opinion, this small but statistically significant difference does not constitute a functionally significant improvement in COT for those with amputation and is likely related to the limited push-off power provided by ESR feet. Further research into both the design of passive prostheses and devices with active push-off is strongly recommended. We also encourage future studies of prosthetic feet to always include nondisabled control subjects because this vastly improves the ability to compare alternative designs and aids in removing potential protocol bias in review articles. 


\section{ACKNOWLEDGMENTS}

\section{Author Contributions:}

Study concept and design: J. Gardiner, A. Z. Bari, D. Howard, L. Kenney.

Acquisition of data: J. Gardiner.

Analysis and interpretation of data: J. Gardiner.

Drafting of manuscript: J. Gardiner, A. Z. Bari, D. Howard, L. Kenney.

Financial Disclosures: The authors have declared that no competing interests exist.

Funding/Support: This material was based on work supported by the Engineering and Physical Sciences Research Council (Swindon, United Kingdom) (grant EP/K019759/1).

Additional Contributions: We would like to thank Robert WeinertAplin and Charlotte Brassey for their helpful discussions regarding the work. We also appreciate the helpful comments of two anonymous reviewers that substantially improved the final manuscript.

\section{REFERENCES}

1. Cruz CP, Eidt JF, Capps C, Kirtley L, Moursi MM. Major lower extremity amputations at a Veterans Affairs hospital. Am J Surg. 2003;186(5):449-54. [PMID:14599605] http://dx.doi.org/10.1016/j.amjsurg.2003.07.027

2. Horgan O, MacLachlan M. Psychosocial adjustment to lower-limb amputation: A review. Disabil Rehabil. 2004; 26(14-15):837-50. [PMID:15497913] http://dx.doi.org/10.1080/09638280410001708869

3. Schmalz T, Blumentritt S, Jarasch R. Energy expenditure and biomechanical characteristics of lower limb amputee gait: The influence of prosthetic alignment and different prosthetic components. Gait Posture. 2002;16(3):255-63. [PMID:12443950] http://dx.doi.org/10.1016/S0966-6362(02)00008-5

4. Nielsen DH, Shurr DG, Golden JC, Meier K. Comparison of energy cost and gait efficiency during ambulation in below-knee amputees using different prosthetic feet - a preliminary report. J Prosthet Orthot. 1988;1(1):24-31. http://dx.doi.org/10.1097/00008526-198810000-00006

5. Genin JJ, Bastien GJ, Franck B, Detrembleur C, Willems PA. Effect of speed on the energy cost of walking in unilateral traumatic lower limb amputees. Eur J Appl Physiol. 2008;103(6):655-63. [PMID:18478251] http://dx.doi.org/10.1007/s00421-008-0764-0

6. Mengelkoch LJ, Kahle JT, Highsmith MJ. Energy costs \& performance of transtibial amputees \& non-amputees during walking \& running. Int J Sports Med. 2014;35(14): 1223-28. [PMID:25144429] http://dx.doi.org/10.1055/s-0034-1382056

7. Waters RL, Perry J, Antonelli D, Hislop H. Energy cost of walking of amputees: The influence of level of amputation.
J Bone Joint Surg Am. 1976;58(1):42-46.

[PMID:1249111]

http://dx.doi.org/10.2106/00004623-197658010-00007

8. Fukunaga T, Kubo K, Kawakami Y, Fukashiro S, Kanehisa $\mathrm{H}$, Maganaris CN. In vivo behaviour of human muscle tendon during walking. Proc Biol Sci. 2001;268(1464):229-33. [PMID:11217891] http://dx.doi.org/10.1098/rspb.2000.1361

9. Fukunaga T, Kawakami Y, Kubo K, Kanehisa H. Muscle and tendon interaction during human movements. Exerc Sport Sci Rev. 2002;30(3):106-10. [PMID:12150568] http://dx.doi.org/10.1097/00003677-200207000-00003

10. Ishikawa M, Komi PV, Grey MJ, Lepola V, Bruggemann G-P. Muscle-tendon interaction and elastic energy usage in human walking. J Appl Physiol. 2005;99(2):603-08. [PMID:15845776] http://dx.doi.org/10.1152/japplphysiol.00189.2005

11. Zelik KE, Huang T-WP, Adamczyk PG, Kuo AD. The role of series ankle elasticity in bipedal walking. J Theor Biol. 2014;346:75-85. [PMID:24365635] http://dx.doi.org/10.1016/j.jtbi.2013.12.014

12. Casillas JM, Dulieu V, Cohen M, Marcer I, Didier JP. Bioenergetic comparison of a new energy-storing foot and SACH foot in traumatic below-knee vascular amputations. Arch Phys Med Rehabil. 1995;76(1):39-44.

[PMID:7811172] http://dx.doi.org/10.1016/S0003-9993(95)80040-9

13. Hsu M-J, Nielsen DH, Yack HJ, Shurr DG. Physiological measurements of walking and running in people with transtibial amputations with 3 different prostheses. J Orthop Sports Phys Ther. 1999;29(9):526-33. [PMID:10518294] http://dx.doi.org/10.2519/jospt.1999.29.9.526

14. Lehmann JF, Price R, Boswell-Bessette S, Dralle A, Questad K. Comprehensive analysis of dynamic elastic response feet: Seattle Ankle/Lite Foot versus SACH foot. Arch Phys Med Rehabil. 1993;74(8):853-61. [PMID:8347071] http://dx.doi.org/10.1016/0003-9993(93)90013-Z

15. Lehmann JF, Price R, Boswell-Bessette S, Dralle A, Questad K, deLateur BJ. Comprehensive analysis of energy storing prosthetic feet: Flex Foot and Seattle Foot versus standard SACH foot. Arch Phys Med Rehabil. 1993; 74(11):1225-31. [PMID:8239969]

16. Hsu M-J, Nielsen DH, Lin-Chan S-J, Shurr D. The effects of prosthetic foot design on physiologic measurements, self-selected walking velocity, and physical activity in people with transtibial amputation. Arch Phys Med Rehabil. 2006;87(1):123-29. [PMID:16401450] http://dx.doi.org/10.1016/j.apmr.2005.07.310

17. Torburn L, Powers CM, Guiterrez R, Perry J. Energy expenditure during ambulation in dysvascular and traumatic below-knee amputees: A comparison of five prosthetic feet. J Rehabil Res Dev. 1995;32(2):111-19.

[PMID:7562650] 
18. Barth DG, Schumacher L, Thomas SS. Gait analysis and energy cost of below-knee amputees wearing six different prosthetic feet. J Prosthet Orthot. 1992;4(2):63-75. http://dx.doi.org/10.1097/00008526-199212000-00001

19. Hofstad C, Linde H, Limbeek J, Postema K. Prescription of prosthetic ankle-foot mechanisms after lower limb amputation. Cochrane Database Syst Rev. 2004;(1):CD003978. [PMID:14974050]

20. Wezenberg D, van der Woude LH, Faber WX, de Haan A, Houdijk H. Relation between aerobic capacity and walking ability in older adults with a lower-limb amputation. Arch Phys Med Rehabil. 2013;94(9):1714-20.

[PMID:23466292]

http://dx.doi.org/10.1016/j.apmr.2013.02.016

21. Hreljac A. Preferred and energetically optimal gait transition speeds in human locomotion. Med Sci Sports Exerc. 1993;25(10):1158-62. [PMID:8231761] http://dx.doi.org/10.1249/00005768-199310000-00012

22. Postema K, Hermens HJ, de Vries J, Koopman HF, Eisma WH. Energy storage and release of prosthetic feet. Part 1: Biomechanical analysis related to user benefits. Prosthet Orthot Int. 1997;21(1):17-27. [PMID:9141122]

23. Segal AD, Orendurff MS, Klute GK, McDowell ML, Pecoraro JA, Shofer J, Czerniecki JM. Kinematic and kinetic comparisons of transfemoral amputee gait using C-Leg and Mauch SNS prosthetic knees. J Rehabil Res Dev. 2006; 43(7):857-70. [PMID:17436172] http://dx.doi.org/10.1682/JRRD.2005.09.0147

24. Ferris AE, Aldridge JM, Rábago CA, Wilken JM. Evaluation of a powered ankle-foot prosthetic system during walking. Arch Phys Med Rehabil. 2012;93(11):1911-18. [PMID:22732369] http://dx.doi.org/10.1016/j.apmr.2012.06.009

25. Herr HM, Grabowski AM. Bionic ankle-foot prosthesis normalizes walking gait for persons with leg amputation.
Proc Biol Sci. 2012;279(1728):457-64. [PMID:21752817]

http://dx.doi.org/10.1098/rspb.2011.1194

26. De Asha AR, Munjal R, Kulkarni J, Buckley JG. Impact on the biomechanics of overground gait of using an 'Echelon' hydraulic ankle-foot device in unilateral trans-tibial and trans-femoral amputees. Clin Biomech (Bristol, Avon). 2014;29(7):728-34. [PMID:24997811] http://dx.doi.org/10.1016/j.clinbiomech.2014.06.009

27. Versluys R, Beyl P, Van Damme M, Desomer A, Van Ham R, Lefeber D. Prosthetic feet: State-of-the-art review and the importance of mimicking human ankle-foot biomechanics. Disabil Rehabil Assist Technol. 2009;4(2):65-75. [PMID:19253096] http://dx.doi.org/10.1080/17483100802715092

Submitted for publication April 14, 2016. Accepted in revised form November 18, 2015.

This article and any supplementary material should be cited as follows:

Gardiner J, Bari AZ, Howard D, Kenney L. Transtibial amputee gait efficiency: Energy storage and return versus solid ankle cushioned heel prosthetic feet. J Rehabil Res Dev. 2016;53(6):1133-38. http://dx.doi.org/10.1682/JRRD.2015.04.0066

ORCID: James Gardiner, PhD: 0000-0003-1902-3416; Laurence Kenney, PhD: 0000-0003-2164-3892

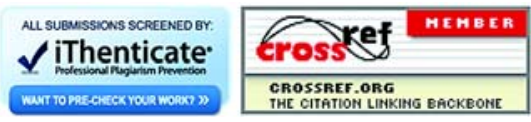

